\title{
ALGEBRAIC THEORY OF CURVETTES AND DICRITICALS
}

\author{
SHREERAM S. ABHYANKAR AND ENRIQUE ARTAL BARTOLO \\ (Communicated by Lev Borisov)
}

Abstract. We relate curvettes with dicriticals.

\section{INTRODUCTION}

The algebraic theory of dicriticals was developed in Ab9 to Ab14, AH1 to AH2, and AL1 to AL2. Now, based on the idea of curvettes, we give a constructive version of the theory. Here we deal with algebraic aspects, with Theorem (3.4) as the main result, and elsewhere we shall deal with analytic aspects.

\section{TERminology}

We shall follow the terminology of Ab8 to Ab14, especially that of Section 2 and Remark (4.0) of Ab11, Sections 2 and 3 of Ab12, Sections 2 to 4 of Ab13, and Sections 2 to 5 of [Ab14. Some other basic references are Ab1 to Ab7]. For any ring $A$ we put

$$
P(A)=\text { set of all nonzero principal ideals in } A
$$

with

$$
P^{\times}(A)=P(A) \backslash\{A\} \text { and } P^{*}(A)=P(A) \cap \operatorname{spec}(A) .
$$

Referring to Section 2 of Ab12] for definitions, for any overring $B$ of $A$ we put

$$
\bar{A}^{B}=\text { integral closure of } A \text { in } B
$$

and we note that $\bar{A}^{B}$ is a subring of $B$, and for any ideal $J$ in $A$ we put

$$
J^{-B}=\text { the integral closure of } J \text { in } B
$$

and we note that $J^{-B}$ is an ideal in $\bar{A}^{B}$.

We recall that if $B$ is quasilocal, then $H_{B}: B \rightarrow H(B)=B / M(B)$ denotes the residue class epimorphism and, letting $\mathrm{QF}$ denote the quotient field, we put

$$
\chi(A, B)=\left[K^{\prime}: K\right]
$$

where $K^{\prime}$ is the algebraic closure of $K=\mathrm{QF}\left(H_{B}(A)\right)$ in $H(B)$.

Now let $R$ be a two dimensional regular local domain with quotient field $L$.

See Section 2 of Ab11 and Section 2 of Ab13 for the definitions of the symbols $D(R)^{\Delta}, D_{j}(R)^{\Delta}, Q(R), Q_{j}(R), Q(R)^{\Delta}, Q_{j}(R)^{\Delta}, \mathfrak{Q}(R, I), \mathfrak{Q}_{j}(R, I), \mathfrak{P}(R, I)$, $\mathfrak{P}_{j}(R, I)$, QDT and $o_{R}, \mathfrak{Q}(R, J), \mathfrak{B}(R, J)$ and $\mathfrak{D}(R, J)=$ dicritical set of $J$ in $R$.

Received by the editors February 1, 2012.

2010 Mathematics Subject Classification. Primary 14A05.

Key words and phrases. Curvette, dicritical divisor, multiplicity sequence.

The second author was partially supported by MTM2010-21740-C02-02. 
See Section 2 of Ab12 for the definitions of simple ideal, complete ideal, normal ideal, valuation ideal, $C(R)=($ the set of all $M(R)$-primary simple complete ideals in $R) \subset \bar{C}(R)=($ the set of all nonzero complete ideals in $R$ ), and the Zariski map $\zeta_{R}: D(R)^{\Delta} \rightarrow C(R)$.

Recall that if $\left(R_{j}\right)_{0 \leq j \leq \nu}$ is the finite QDT sequence of $R$ along $V \in D(R)^{\Delta}$, then $o_{R}^{-1}(V)=R_{\nu}$. By analogy, for any $T \in Q_{1}\left(R_{\nu}\right)$ we put $o_{R}^{*}(T)=R_{\nu}$, and we put

$$
\pi(R, V)=Q_{1}\left(R_{\nu}\right)=Q_{1}\left(o_{R}^{-1}(V)\right)=\text { set of all points of } V
$$

and

$$
\left\{\begin{aligned}
\pi^{*}(R, V) & =\left\{T \in \pi(R, V): T \not \subset o\left(R_{j}\right) \text { for } 0 \leq j<\nu\right\} \\
& =\text { set of all free points of } V .
\end{aligned}\right.
$$

For any $T \in \pi(R, V)$ with $V \in D(R)^{\Delta}$ we put

$$
\xi(R, T, V)=T \cap M(V)=\text { the parameter ideal of }(R, T, V)
$$

and we observe that then we have $\xi(R, T, V) \in P^{*}(T)$ with $\operatorname{ord}_{T} \xi(R, T, V)=1$ and $V=T_{\xi(R, T, V)}$. Then we put

$$
\gamma(R, T, V)=\left\{\begin{array}{l}
\left\{\mathfrak{c} \in P^{\times}(T): \operatorname{ord}_{T} \mathfrak{c}=\operatorname{ord}_{(T / \xi(R, T, V))} \mathfrak{c}\right\} \\
=\text { set of all } \operatorname{arcs} \text { of }(R, T, V)
\end{array}\right.
$$

and we define the weight of $\mathfrak{c}$ by letting

$$
\theta(R, T, \mathfrak{c})=\chi\left(o_{R}^{*}(T), T\right) \operatorname{ord}_{T} \mathfrak{c} .
$$

We put

$$
\gamma^{*}(R, T, V)=\left\{\begin{array}{l}
\gamma(R, T, V) \text { or } \emptyset \text { according as } T \in \pi^{*}(R, V) \text { or } T \notin \pi^{*}(R, V) \\
=\text { set of all curvettes of }(R, T, V) .
\end{array}\right.
$$

Next we put:

$\sigma(R)=$ set of all stems of $R$, i.e., all finite sequences $S=\left(S_{j}, I_{j}\right)_{0 \leq j \leq \mu}$ where $\left(S_{j}\right)_{0 \leq j \leq \mu}$ is a finite QDT sequence of $R$ with $I_{j} \in P^{\times}\left(R_{j}\right)$ for $0 \leq j \leq \mu$, such that for $0 \leq j<\mu$ we have $\left(S_{j}, S_{j+1}\right)\left(I_{j}\right)=I_{j+1}$, and $\left(S_{j}, S_{j+1}^{\prime}\right)\left(I_{j}\right)=S_{j+1}^{\prime}$ whenever $S_{j+1}^{\prime} \in Q_{1}\left(S_{j}\right) \backslash\left\{S_{j+1}\right\}$.

For any $V \in D(R)^{\Delta}$ we put:

$\sigma(R, V)=$ set of all arc stems of $(R, V)$, i.e., all members $S=\left(S_{j}, I_{j}\right)_{0 \leq j \leq \mu}$ of $\sigma(R)$ such that $\left(S_{j}\right)_{0 \leq j \leq \mu-1}$ is the finite QDT sequence of $R$ along $V$ with $I_{\mu} \in \gamma\left(R, S_{\mu}, V\right)$. We define the weight of $S$ by letting

$$
\theta(R, S)=\theta\left(R, S_{\mu}, I_{\mu}\right)
$$

and we put

$$
\sigma^{*}(R, V)=\left\{\begin{array}{l}
\left\{S=\left(S_{j}, I_{j}\right)_{0 \leq j \leq \mu} \text { in } \sigma(R, V): I_{\mu} \in \gamma^{*}\left(R, S_{\mu}, V\right)\right\} \\
=\text { set of all curvette stems of }(R, V),
\end{array}\right.
$$

where we call $\mu$ the layer index of $V$ over $R$ and denote it by $\mu(R, V)$, and we note that $V \in D_{\mu-1}(R)^{\Delta}$. 


\section{STUDY OF STEMS}

Let $R$ be a two dimensional regular local domain with quotient field $L$.

Theorem 3.1. Let $(x, y)$ be generators of $M(R)$ and let $\kappa$ be a coefficient set of $R$. Let $S=\left(S_{j}, x_{j}, y_{j}, \kappa_{j}\right)_{0 \leq j \leq \nu}$ be any finite $Q D T$ sequence of $(R, x, y, \kappa)$. Let us recall that, as in Section 5 of [Ab14], a positive integer $j \leq \nu$ is said to be an $X$-operation or $Y$-operation or a translation of $S$ according as

$$
\left\{\begin{array}{l}
\left(x_{j}, y_{j}\right)=\left(x_{j-1}, y_{j-1} / x_{j-1}\right) \\
\text { or }\left(x_{j}, y_{j}\right)=\left(x_{j-1} / y_{j-1}, y_{j-1}\right) \\
\text { or }\left(x_{j-1}, y_{j-1} / x_{j-1}\right) \neq\left(x_{j}, y_{j}\right) \neq\left(x_{j-1} / y_{j-1}, y_{j-1}\right) .
\end{array}\right.
$$

In (4.6.3) of AL2 the multiplicity sequence $m^{*}(S, 1,1)=m_{j}^{*}(S, 1,1)_{0 \leq j \leq \nu}$ was defined where $m_{j}^{*}(S, 1,1)$ are positive integers such that

$$
m_{0}^{*}(S, 1,1) \geq m_{1}^{*}(S, 1,1) \geq \cdots \geq m_{\nu}^{*}(S, 1,1)=1 .
$$

As was observed in AL2, the sequence $m^{*}(S, 1,1)$ depends only on the "word" $W=W_{1} W_{2} \ldots W_{\nu}$ in the free group on three generators $X, Y, Z$ given by $W_{j}=X$ or $Y$ or $Z^{\chi\left(S_{j}\right)}$ according as $j$ is an $X$-operation or $Y$-operation or a translation of $S$. The following result (3.1.1) was proved in Theorem (4.9) of [AL2, where $V=o\left(S_{\nu}\right)$.

(3.1.1) For any member $\bar{S}=\left(\bar{S}_{j}, I_{j}\right)_{0 \leq j \leq \mu}$ of $\sigma^{*}(R, V)$, with $\nu=\mu-1$ and $\bar{S}_{j}=S_{j}$ for $0 \leq j \leq \nu$, we have

$$
\operatorname{ord}_{S_{j}} I_{j}=m_{j}^{*}(S, 1,1) \theta(R, \bar{S}) \quad \text { for } \quad 0 \leq j \leq \nu .
$$

(3.1.2) Consequently, for any members $\bar{S}=\left(\bar{S}_{j}, I_{j}\right)_{0 \leq j \leq \mu}$ and $\bar{S}^{\prime}=\left(\bar{S}_{j}^{\prime}, I_{j}^{\prime}\right)_{0 \leq j \leq \mu}$ of $\sigma^{*}(R, V)$, with $\nu=\mu-1$ and $\bar{S}_{j}=\bar{S}_{j}^{\prime}=S_{j}$ for $0 \leq j \leq \nu$, upon letting $d_{j}=\operatorname{ord}_{S_{j}} I_{j}$ and $d_{j}^{\prime}=\operatorname{ord}_{S_{j}} I_{j}^{\prime}$, we have

$$
\theta\left(R, \bar{S}^{\prime}\right) d_{j}=\theta(R, \bar{S}) d_{j}^{\prime} \quad \text { for } \quad 0 \leq j \leq \nu .
$$

Remark 3.2. Observe that the system $(x, y, \kappa)$ was used in assertion (3.1.1), and then assertion (3.1.1) was used to prove assertion (3.1.2), which itself does not refer to the system $(x, y, \kappa)$. In other words, to prove (3.1.2) we fixed some generators $(x, y)$ of $M(R)$ and some coefficient set of $R$. This was like fixing a coordinate system for proving some geometric property of a geometric figure in the plane and afterwards wiping out the coordinate system.

Definition 3.3. Given any finite sequence $\left(V_{1}, \ldots, V_{r}\right)$ of (not necessarily distinct) members of $D(R)^{\Delta}$, by a double curvette of $\left(R, V_{1}, \ldots, V_{r}\right)$ we mean a sequence $\left(S_{i j}, I_{i j}, S_{i j}^{\prime}, I_{i j}^{\prime}\right)_{1 \leq i \leq r, 0 \leq j \leq \mu_{i}}$ where, for $1 \leq i \leq r$,

$$
S_{i}=\left(S_{i j}, I_{i j}\right)_{0 \leq j \leq \mu_{i}} \quad \text { and } \quad S_{i}^{\prime}=\left(S_{i j}^{\prime}, I_{i j}^{\prime}\right)_{0 \leq j \leq \mu_{i}}
$$

are members of $\sigma^{*}\left(R, V_{i}\right)$ with $\theta\left(R, S_{i}\right)=\theta\left(R, S_{i}^{\prime}\right)$, satisfying the condition $(\bullet)$

$$
\left(\bigcup_{1 \leq i \leq r}\left\{S_{i \mu_{i}}\right\}\right) \cap\left(\bigcup_{1 \leq i \leq r} Q\left(S_{i \mu_{i}}^{\prime}\right)\right)=\emptyset=\left(\bigcup_{1 \leq i \leq r}\left\{S_{i \mu_{i}}^{\prime}\right\}\right) \cap\left(\bigcup_{1 \leq i \leq r} Q\left(S_{i \mu_{i}}\right)\right),
$$

which can be verbalized by saying that the tops of the $S_{i}$ do not contain (are not QDTs of) the tops of the $S_{j}^{\prime}$ and vice versa. [In a picturesque language, we may 
say that the $r$ chariots (or snakes) $\left(S_{i}, S_{i}^{\prime}\right)$ are not permitted to cut each other (or bite each other) as in the Hollywood movie Ben Hur.]

Theorem 3.4. Let $\left(V_{1}, \ldots, V_{r}\right)$ with $r \in \mathbb{N}_{+}$be any finite sequence of (not necessarily distinct) members of $D(R)^{\Delta}$, and let $\left(S_{i j}, I_{i j}, S_{i j}^{\prime}, I_{i j}^{\prime}\right)_{1 \leq i \leq r, 0 \leq j \leq \mu_{i}}$ be any double curvette of $\left(R, V_{1}, \ldots, V_{r}\right)$. Then upon letting

$$
\mathfrak{a}=\prod_{1 \leq i \leq r} I_{i 0} \quad \text { and } \quad \mathfrak{a}^{\prime}=\prod_{1 \leq i \leq r} I_{i 0}^{\prime}
$$

we have that the ideal $\mathfrak{a}+\mathfrak{a}^{\prime}$ is $M(R)$-primary and

$$
\left(\mathfrak{a}+\mathfrak{a}^{\prime}\right)^{-R}=\prod_{1 \leq i \leq r} \zeta_{R}\left(V_{i}\right)^{\theta\left(R, S_{i}\right)}
$$

with

$$
\mathfrak{D}\left(R, \mathfrak{a}+\mathfrak{a}^{\prime}\right)=\left\{V_{1}, \ldots, V_{r}\right\} .
$$

Proof. Follows from (3.1).

Remark 3.5. It is conceivable that the above "follows from" may not be so obvious to a reader who is not familiar with resolving singularities of plane curves by a sequence of QDTs = Quadratic Transformations à la Max Noether [Noe, who was regarded as the father of algebraic geometry until we learned better from Felix Klein [Kle, who declared that he and his friends Max Noether and Paul Gordon (the King of Invariant Theory Gor]) learned algebraic geometry from George Salmon's book Sal Higher Plane Curves, published in 1852 in Dublin, Ireland. So to be user-friendly, referring to pages 131-141 of Ab6] for the geometry of it, here is a long-winded paraphrase of the "follows from" carried out in a discursive manner.

(1) Notation. In Section 2 there are two displayed boldfaced definitions of weights $\theta$. The second boldfaced weight is sandwiched between the boldfaced definitions of stems $\sigma(R)$, arc stems $\sigma(R, V)$, and curvette stems $\sigma^{*}(R, V)$ with $\sigma^{*}(R, V) \subset$ $\sigma(R, V) \subset \sigma(R)$.

Let $S=\left(S_{j}, I_{j}\right)_{0 \leq j \leq \mu}$ be a member of $\sigma(R)$ with $\mu>0$. The stem property says that, for $0 \leq j<\mu$, the ideal $I_{j}$ is unitangent; i.e., it goes through exactly one member of $Q_{1}\left(S_{j}\right)$

To explain unitangency, following the principle of coordinate fixing enunciated in (3.2), fix any generators $(x, y)$ of $M(R)$ and any coefficient set $\kappa$ of $R$. By (3.1) we get to the heart of our proof saying that

$$
\left\{\begin{array}{l}
\text { fixing generators } F_{i j} \text { and } F_{i j}^{\prime} \text { of the ideals } I_{i j} \text { and } I_{i j}^{\prime} \text { in } S_{i} \\
\text { and letting } D_{i j}=\operatorname{ord}_{S_{i}} F_{i j} \text { and } D_{i j}^{\prime}=\operatorname{ord}_{S_{i}^{\prime}} F_{i j}^{\prime}, \\
\text { we have } D_{i j}=D_{i j}^{\prime} \text { for } 1 \leq i \leq r \text { and } 0 \leq j \leq \nu_{i} \\
\text { where } \nu_{i}=\mu_{i}-1 \text { for } 1 \leq i \leq r .
\end{array}\right.
$$

Now for any $F \in M(R)^{d} \backslash M(R)^{d+1}$ with $d \in \mathbb{N}$, as in (4.0) of Ab11, we have that the initial form info $(F)=\operatorname{info}_{(R, x, y)} F$ is a nonzero homogeneous polynomial of degree $d$ in

$$
\operatorname{grad}(R)=K[X, Y]=\text { the polynomial ring in indeterminates } X, Y
$$

over $K=H(R)$. We write $\operatorname{info}(F R)$ for the ideal in $k[X, Y]$ generated by $\operatorname{info}(F)$ and call this the initial ideal of the ideal $F R$. We write $\operatorname{rad}(\operatorname{info}(F R))$ for the 
radical of info $(F R)$ in $K[X, Y]$. We say that $F$ and $F R$ are unitangent, meaning that $\operatorname{rad}(\operatorname{info}(F R))$ is a prime ideal in $K[X, Y]$. Note that then $F R$ is unitangent iff info $(F R)=\phi^{e} K[X, Y]$ for some $e \in \mathbb{N}_{+}$and some homogeneous irreducible $\phi \in K[X, Y] \backslash K$. Also note that $F R$ is unitangent iff there is exactly one member $R^{\prime}$ of $Q_{1}(R)$ for which $\left(R, R^{\prime}\right)(F R) \neq R^{\prime}$. Without assuming it to be unitangent, by info $(F)^{*}$ and $\operatorname{info}(F R)^{*}$ we denote the unique generator of $\operatorname{info}(F R)$, which is of the form $X^{p} Y^{q}+($ terms of $Y$-degree $<q)$.

For the sake of completeness, if $F=0$, then we put info $(F)=\operatorname{info}_{(R, x, y)} F=0$ and $\operatorname{rad}(\operatorname{info}(F R))=\operatorname{info}(F R)=\{0\}$.

Given any UFD $A$ and given any $\Phi=$ a subset of $A$ or $\Phi=$ a set of subsets of $A$, by $\operatorname{GCD}(\Phi)_{A}$ we denote the smallest nonzero principal ideal $\Psi$ in $A$ such that $\phi \in \Psi$ or $\phi \subset \Psi$ for all $\phi \in \Phi$. We write $\operatorname{GCD}(\Phi)_{A}=1$ to mean $\operatorname{GCD}(\Phi)_{A}=A$, and we write $\operatorname{GCD}(\Phi)_{A} \neq 1$ to mean $\operatorname{GCD}(\Phi)_{A} \neq A$. In dealing with the case of $A=\operatorname{grad}(R)=K[X, Y]$, we may write $\operatorname{GCD}(\Phi)_{A}^{*}$ for the concrete or starred GCD, by which we mean the unique generator of the ideal $\operatorname{GCD}(\Phi)_{A}$ which is of the form $X^{p} Y^{q}+($ terms of $Y$-degree $<q)$, and we may drop the subscript $A$. We are mostly interested in situations like $\Phi=\left\{\operatorname{info}\left(I_{1}\right)\right.$, info $\left.\left(I_{2}\right)\right\}$ with $I_{1}, I_{2}$ in $P^{\times}(R)$, and then we may write $\operatorname{GCD}\left(\operatorname{info}\left(I_{1}\right), \operatorname{info}\left(I_{2}\right)\right)^{*}$ for $\operatorname{GCD}(\Phi)_{A}^{*}$.

$(*)$ Note that by convention on empty or infinite sums or products: empty sums are zero, empty products are one (or a unit ideal), terms reduced to zero in an infinite sum are disregarded, and terms reduced to one (or a unit ideal) in an infinite product are disregarded.

(2) Zariski number. From the beginning of Section 2 of Ab13, recall the definitions of things such as the ideal tree $\mathfrak{Q}(R, J)$ and singularity tree $\mathfrak{P}(R, J)$ of a nonzero ideal $J$ in $R$. Moreover, let us introduce the Zariski number $m(R, J)$, which is a nonnegative integer obtained by putting

$$
m(R, J)=d-s
$$

where $d=\operatorname{ord}_{R} J$ and $s$ is the $(X, Y)$-degree of the GCD* of the initial forms of all the elements of $J$ of $R$-order $d$. Furthermore, for any $T \in Q(R)$ we introduce the derived Zariski number $m(R, J, T)$, which is a nonnegative integer obtained by putting

$$
m(R, J, T)=m\left(T, J_{T}\right) \quad \text { where } \quad J_{T}=(R, T)(J) .
$$

Finally, for any $V \in D(R)^{\Delta}$ we introduce the Zariski index $n(R, J, V)$, which is a nonnegative integer obtained by putting

$$
n(R, J, V)=m\left(R, J, o_{R}^{-1}(V)\right) .
$$

Referring to convention $(*)$, the famous Zariski Factorization Theorem proved in Appendix 5 of [ $\mathrm{ZaS}$ ], and restated in (2.4) of [Ab12], can be paraphrased by saying that

$$
J^{-R}=\operatorname{GCD}(J)_{R} \prod_{V \in D(R)^{\Delta}} \zeta_{R}(V)^{n(R, J, V)}
$$

and by noting that the finite set $\mathfrak{D}(R, J)$ is characterized by saying

$$
\mathfrak{D}(R, J)=\left\{V \in D(R)^{\Delta}: n(R, J, V)>0\right\} .
$$

Again referring to convention $(*)$, we claim that (with $D_{i 0}$ defined in $(\sharp)$ )

$$
m\left(R, \mathfrak{a}+\mathfrak{a}^{\prime}\right)=\sum_{i \in B(R)} D_{i 0} \text { where } B(R)=\left\{1 \leq i \leq r: V_{i}=o(R)\right\} .
$$


Proof. Relabelling $V_{1}, \ldots, V_{r}$ suitably we can find an integer $p$ with $0 \leq p \leq r$ such that

$$
V_{i} \neq o(R) \text { for } 1 \leq i \leq p
$$

and

$$
V_{i}=o(R) \text { for } p<i \leq r .
$$

Let

$$
J=\mathfrak{a}+\mathfrak{a}^{\prime} \text { with } F=F_{10} \ldots F_{r 0} \text { and } F^{\prime}=F_{10}^{\prime} \ldots F_{r 0}^{\prime} .
$$

By $(\sharp)$ we have

$$
J=F R+F^{\prime} R \text { with } \operatorname{ord}_{R} F_{0 i}=D_{i 0}=\operatorname{ord}_{R} F_{i 0}^{\prime} \text { for } 1 \leq i \leq r,
$$

and hence upon letting

$$
d=\sum_{1 \leq i \leq r} D_{i 0} \text { and } s=\sum_{1 \leq i \leq p} D_{i 0}
$$

we get

$$
\operatorname{ord}_{R} F=\operatorname{ord}_{R} J=d=\operatorname{ord}_{R} F^{\prime}
$$

and

Therefore

$$
\operatorname{ord}_{R}\left(F_{10} \ldots F_{p 0}\right)=s=\operatorname{ord}_{R}\left(F_{10}^{\prime} \ldots F_{p 0}^{\prime}\right)
$$

the RHS of $(\dagger)$ equals $d-s$.

For $1 \leq i \leq p$, by definition $\nu_{i} \geq 1$ with $S_{i 1}=S_{i 1}^{\prime}$, and hence by the stem property we see that $F_{i 0}$ and $F_{i 0}^{\prime}$ are unitangent with info $\left(F_{i 0} R\right)=\operatorname{info}\left(F_{i 0}^{\prime} R\right)$; consequently

$$
\left\{\begin{array}{l}
\operatorname{info}\left(F_{10} \ldots F_{p 0}\right)^{*}=\operatorname{info}\left(F_{10}^{\prime} \ldots F_{p 0}^{\prime}\right)^{*} \text { and this common info } \\
\text { divides the info of every element of } J \text { of } R \text {-order } d .
\end{array}\right.
$$

By $(\bullet)$ we see that

$$
S_{i 1} \not \subset S_{i^{\prime} \mu_{i^{\prime}}}^{\prime} \text { for } p<i \leq r \text { and } 1 \leq i^{\prime} \leq r
$$

and

therefore

$$
S_{i^{\prime} 1}^{\prime} \not \subset S_{i \mu_{i}} \text { for } p<i^{\prime} \leq r \text { and } 1 \leq i \leq r \text {; }
$$

$$
\operatorname{GCD}\left(\operatorname{info}\left(F_{i 0} R\right), \operatorname{info}\left(F_{i^{\prime} 0}^{\prime} R\right)\right)=1 \text { for } p<i \leq r \text { and } 1 \leq i^{\prime} \leq r
$$

and

$$
\operatorname{GCD}\left(\operatorname{info}\left(F_{i^{\prime} 0}^{\prime} R\right), \operatorname{info}\left(F_{i 0} R\right)\right)=1 \text { for } p<i^{\prime} \leq r \text { and } 1 \leq i \leq r .
$$

Hence $\operatorname{info}\left(F_{10} \ldots F_{p 0}\right)^{*}$ is the GCD* of the initial forms of all the elements of $J$ of $R$-order $d$, and the $(X, Y)$-degree of $\operatorname{info}\left(F_{10} \ldots F_{p 0}\right)^{*}$ clearly equals $s$. Therefore

$$
\text { the LHS of }(\dagger) \text { equals } d-s \text {. }
$$

Thus the LHS and the RHS of $(\dagger)$ both equal $d-s$, and so we are done.

(3) Primariness. Let us prove a somewhat more general version of the part of (3.4) which asserts that the ideal $\mathfrak{a}+\mathfrak{a}^{\prime}$ is $M(R)$-primary. For inductive purposes we introduce the chopped version $\bar{S}=\left(\bar{S}_{j}, \bar{I}_{j}\right)_{0 \leq j \leq \bar{\mu}}$ of a member $S=\left(S_{j}, I_{j}\right)_{0 \leq j \leq \mu}$ of $\sigma(R)$ with $\mu>0$ by putting $\bar{\mu}=\mu-1$ and $\left(\bar{S}_{j}, \bar{I}_{j}\right)=\left(S_{j+1}, I_{j+1}\right)$ for $0 \leq j \leq \bar{\mu}$. Note that $\bar{S}$ is a member of $\sigma\left(S_{1}\right)$. Also note that since $I_{0}$ and $I_{0}^{\prime}$ are nonzero nonunit principal ideals in $R$, we have

$$
\operatorname{GCD}\left(I_{0}, I_{0}^{\prime}\right)_{R}=1 \Leftrightarrow I_{0}+I_{0}^{\prime} \text { is } M(R) \text {-primary. }
$$


We claim that given any other member $S^{\prime}=\left(S_{j}^{\prime}, I_{j}^{\prime}\right)_{0 \leq j \leq \mu^{\prime}}$ of $\sigma(R)$ with $\mu^{\prime}>0$ we have the following assertions.

Claim (i). If $\operatorname{GCD}\left(I_{0}, I_{0}^{\prime}\right) \neq 1$, then $S_{1}=S_{1}^{\prime}$ and $\operatorname{GCD}\left(I_{1}, I_{1}^{\prime}\right)_{S_{1}} \neq 1$.

Claim (ii). If $S_{\mu} \not \subset S_{\mu^{\prime}}^{\prime} \not \subset S_{\mu}$, then $\operatorname{GCD}\left(I_{0}, I_{0}^{\prime}\right)=1$.

To prove (i), assume that there exist nonzero principal ideals $J, I^{*}, I^{\prime *}$ in $R$ with $J \neq R$ such that $I_{0}=J I^{*}$ and $I_{0}^{\prime}=J I^{\prime *}$. Now there exists $T \in \mathfrak{P}_{1}(R, J)$, and for any such $T$ we have $T \in \mathfrak{P}_{1}\left(R, I_{0}\right) \cap \mathfrak{P}_{1}\left(R, I_{0}^{\prime}\right)$; hence $S_{1}=S_{1}^{\prime}$ because the stem property implies $\mathfrak{P}_{1}\left(R, I_{0}\right)=\left\{S_{1}\right\}$ and $\mathfrak{P}_{1}\left(R, I_{0}^{\prime}\right)=\left\{S_{1}^{\prime}\right\}$. Now upon letting $J_{1}=\left(R, S_{1}\right)(J)$ with $I_{1}^{*}=\left(R, S_{1}\right)\left(I^{*}\right)$ and $I_{1}^{\prime *}=\left(R, S_{1}\right)\left(I^{\prime *}\right)$, we see that $J_{1}, I_{1}^{*}, I_{1}^{\prime *}$ are nonzero principal ideals in $S_{1}$ with $J_{1} \neq S_{1}$ such that $I_{1}=J_{1} I_{1}^{*}$ and $I_{1}^{\prime}=J_{1} I_{1}^{\prime *}$. Therefore $\operatorname{GCD}\left(I_{1}, I_{1}^{\prime}\right)_{S_{1}} \neq 1$.

To prove (ii), assume $S_{\mu} \not \subset S_{\mu^{\prime}}^{\prime} \not \subset S_{\mu}$. By induction on $\min \left(\mu, \mu^{\prime}\right)$ we shall show that $\operatorname{GCD}\left(I_{0}, I_{0}^{\prime}\right)=1$. If $\min =1$, then we are done by (i). So let $\min >1$ and assume the result is true for all smaller values of min. If $\operatorname{GCD}\left(I_{0}, I_{0}^{\prime}\right)_{R}=1$, then we are done. So assume that $\operatorname{GCD}\left(I_{0}, I_{0}^{\prime}\right)_{R} \neq 1$. Then by (i) we see that $S_{1}=S_{1}^{\prime}$ and $\operatorname{GCD}\left(I_{1}, I_{1}^{\prime}\right)_{S_{1}} \neq 1$. Now $\bar{S}$ and $\bar{S}^{\prime}$ are members of $\sigma\left(S_{1}\right)$ with $0<\min \left(\bar{\mu}, \bar{\mu}^{\prime}\right)<\min \left(\mu, \mu^{\prime}\right)$ and $\bar{S}_{\bar{\mu}} \not \subset \bar{S}_{\bar{\mu}^{\prime}}^{\prime} \not \subset \bar{S}_{\bar{\mu}}$. Consequently by the induction hypothesis we get $\operatorname{GCD}\left(I_{1}, I_{1}^{\prime}\right)_{S_{1}}=1$, which is a contradiction. This completes the proof of (ii).

Now we shall prove the following claim, which is obviously more general than the $M(R)$-primary part of (3.4).

Claim (iii). Let $r, r^{\prime}, \mu_{1}, \ldots, \mu_{r}, \mu_{1}^{\prime}, \ldots, \mu_{r^{\prime}}^{\prime}$ be positive integers. For $1 \leq i \leq r$ and $1 \leq i^{\prime} \leq r^{\prime}$ let

$$
S_{i}=\left(S_{i j}, I_{i j}\right)_{0 \leq j \leq \mu_{i}} \quad \text { and } \quad S_{i^{\prime}}^{\prime}=\left(S_{i^{\prime} j}^{\prime}, I_{i^{\prime} j}^{\prime}\right)_{0 \leq j \leq \mu_{i^{\prime}}^{\prime}}
$$

be members of $\sigma(R)$ satisfying the condition $\left(\bullet^{\prime}\right)$

$$
\left(\bigcup_{1 \leq i \leq r}\left\{S_{i \mu_{i}}\right\}\right) \cap\left(\bigcup_{1 \leq i^{\prime} \leq r^{\prime}} Q\left(S_{i^{\prime} \mu_{i^{\prime}}}^{\prime}\right)\right)=\emptyset=\left(\bigcup_{1 \leq i^{\prime} \leq r^{\prime}}\left\{S_{i^{\prime} \mu_{i^{\prime}}}^{\prime}\right\}\right) \cap\left(\bigcup_{1 \leq i \leq r} Q\left(S_{i \mu_{i}}\right)\right) .
$$

Let

$$
\mathfrak{a}=\prod_{1 \leq i \leq r} I_{i 0} \quad \text { and } \quad \mathfrak{a}^{\prime}=\prod_{1 \leq i^{\prime} \leq r^{\prime}} I_{i^{\prime} 0}^{\prime}
$$

Then the ideal $\mathfrak{a}+\mathfrak{a}^{\prime}$ is $M(R)$-primary.

To prove (iii), it suffices to note that, by (ii), the nonzero principal ideals $I_{i 0}$ and $I_{j 0}^{\prime}$ in $R$ have no nonzero nonunit common factor in $R$, and hence neither do their products $\mathfrak{a}$ and $\mathfrak{a}^{\prime}$. Therefore the ideal $\mathfrak{a}+\mathfrak{a}^{\prime}$ is $M(R)$-primary.

The deviation of notation, where $\sigma^{*}(R, V)$ was replaced by the bigger set $\sigma(R)$ and condition $(\bullet)$ was replaced by its weaker version $\left(\bullet^{\prime}\right)$, was only for (iii), and henceforth we revert to the notation of (3.4). At any rate, having established the $M(R)$-primary part of (3.4), we may visualize $R$ to be the local ring of a simple point of a surface (which may be algebraic or analytic or arithmetical), and we may visualize $\mathfrak{a}$ and $\mathfrak{a}^{\prime}$ as curves on that surface passing through that point but having no common component there. 
(4) Chopping. Let

$$
J=\mathfrak{a}+\mathfrak{a}^{\prime} \text { and } J_{T}=(R, T)(J) \text { for all } T \in Q(R) .
$$

Then, referring to convention $\left(^{*}\right)$, as another application of the chopping principle, we claim that for any $T \in Q_{l}(R)$ with $l \in \mathbb{N}$ we have

$$
m(R, J, T)=\sum_{i \in B(T)} D_{i l} \text { where } B(T)=\left\{1 \leq i \leq r: V_{i}=o(T)\right\} .
$$

Proof. Induction on $l$. For $l=0$ we are reduced to $(\dagger)$. So let $l>0$ and assume the result is true for all smaller values of $l$. Let $\bar{R}$ be the unique member of $Q_{1}(R)$ such that $T \in Q_{l-1}(\bar{R})$. Let

$$
E=\left\{S_{i j}: 1 \leq i \leq r \text { with } j=\mu_{i}\right\} \text { and } E^{\prime}=\left\{S_{i j}^{\prime}: 1 \leq i \leq r \text { with } j=\mu_{i}\right\}
$$

and consider the nonzero principal ideals $\bar{I}$ and $\bar{I}^{\prime}$ in $T$ given by

$$
\bar{I}=\prod_{i \in C} I_{i 1} \quad \text { where } \quad C=\left\{1 \leq i \leq r: \bar{R}=S_{i 1}\right\}
$$

and

$$
\bar{I}^{\prime}=\prod_{i \in C^{\prime}} I_{i 1}^{\prime} \quad \text { where } \quad C^{\prime}=\left\{1 \leq i \leq r: \bar{R}=S_{i 1}^{\prime}\right\} .
$$

By $(\sharp)$ it follows that

$$
J_{T}=\bar{I}+\bar{I}^{\prime} .
$$

If $\bar{R} \in E$, then by $(\bullet)$ we see that $B(R)=\emptyset=C^{\prime}$, and hence the RHS of ( $\left.\ddagger\right)$ equals zero and $\bar{I}^{\prime}=T$, and hence the LHS of ( $\left.\ddagger\right)$ equals zero by $\left(\sharp^{*}\right)$. If $\bar{R} \in E^{\prime}$, then by $(\bullet)$ we see that $B(R)=\emptyset=C$, and hence the RHS of ( $)$ equals zero and $\bar{I}=T$, and hence the LHS of $(\ddagger)$ equals zero by $\left(\sharp^{*}\right)$. So now assume that $\bar{R} \notin E \cup E^{\prime}$. Then

$$
C=C^{\prime}=\left\{1 \leq i \leq r: \nu_{i}>0 \text { and } \bar{R}=S_{i 1}=S_{i 1}^{\prime}\right\} .
$$

It follows that if $C=\emptyset$, then both sides of $(\ddagger)$ are zero. Also assume that $C \neq \emptyset$.

Now clearly we are in the $l-1$ case. In greater detail, for every $i \in C$ let

$$
\bar{S}_{i}=\left(\bar{S}_{i j}, \bar{I}_{i j}\right)_{0 \leq j \leq \bar{\mu}_{i}} \text { and } \bar{S}_{i}^{\prime}=\left(\bar{S}_{i j}^{\prime}, \bar{I}_{i j}^{\prime}\right)_{0 \leq j \leq \bar{\mu}_{i}},
$$

with $\bar{\mu}_{i}=\mu_{i}-1$ being the chopped versions of $S_{i}$ and $S_{i}^{\prime}$ respectively. Also let

$$
\overline{\mathfrak{a}}=\prod_{1 \leq i \leq r} \bar{I}_{i 0} \quad \text { and } \quad \overline{\mathfrak{a}}^{\prime}=\prod_{1 \leq i \leq r} \bar{I}_{i 0}^{\prime}
$$

By $\left(\sharp^{*}\right)$ we get $J_{\bar{R}}=\overline{\mathfrak{a}}+\overline{\mathfrak{a}}^{\prime}$, and clearly

$$
m(R, J, T)=m\left(\bar{R}, J_{\bar{R}}, T\right)
$$

and

$$
\sum_{i \in B(T)} D_{i l}=\sum_{\left\{i: i \in C \text { with } V_{i}=o(T)\right\}} \operatorname{ord}_{\bar{R}} \bar{I}_{i, l-1},
$$

and condition $(\bar{\bullet})$ which is obtained from $(\bullet)$ by an obvious change of notation is satisfied. Since $T \in Q_{l-1}(\bar{R})$, we are done by the induction hypothesis. 
(5) Basic fact. Clearly (3.4.2) follows from (3.4.1). In (3.4.1), why is the Zariski simple ideal $\zeta_{R}\left(V_{i}\right)$ raised to the power $\theta\left(R, S_{i}\right)$ ? This is dictated by items $(2 \mathrm{~A})$ to (2D) of Ab13], where the geometrically worded pages 131-141 of [Ab6] are put on a firm algebraic foundation by proving the following basic fact from the resolution of singularities, which is actually included in (3.1):

Fixing any positive integer $i \leq r$, let

$$
\left(T, I, T^{*}, I^{*}\right)=\left(S_{i, \mu_{i}}, I_{i, \mu_{i}}, S_{i, \mu_{i}-1}, I_{i, \mu_{i}-1}\right) \text { and }\left(d, d^{*}\right)=\left(\operatorname{ord}_{T} I, \operatorname{ord}_{T^{*}} I^{*}\right) .
$$

Then, speaking in a geometric manner, for the multiplicity $d$ of the transform $I=\left(T^{*}, T\right)\left(I^{*}\right)$ of the "unitangent curve" $I^{*}$ at the "point" $T^{*}$ of multiplicity $d^{*}$, we have $\chi\left(T^{*}, T\right) d<d^{*}$ or $\chi\left(T^{*}, T\right) d=d^{*}$ according as $I$ is or is not tangent to the exceptional line. Therefore, only assuming $S_{i} \in \sigma\left(R, V_{i}\right)$ but without assuming $S_{i} \in \sigma^{*}\left(R, V_{i}\right)$, we get $\theta\left(R, S_{i}\right)=d^{*}$.

(6) Conclusion. Theorem (3.4) follows from $(\sharp),(b)$, Claim (iii), ( $\ddagger)$, and (5). We shall now give a slightly different arrangement of the proof in which Theorem (3.4) is replaced by Theorems (3.6) and (3.7), which are formally independent of the material from $(2.1)$ to $(3.5)$.

Theorem 3.6. Let $\left(V_{1}, \ldots, V_{r}\right)$ be any finite sequence of (not necessarily distinct) members of $D(R)^{\Delta}$ where $r$ is a positive integer, and let there be any double stem of $\left(R, V_{1}, \ldots, V_{r}\right)$, by which we mean a sequence

$$
\left(S_{i j}, I_{i j}, S_{i j}^{\prime}, I_{i j}^{\prime}\right)_{1 \leq i \leq r, 0 \leq j \leq \mu_{i}}
$$

where, for $1 \leq i \leq r$,

$$
S_{i}=\left(S_{i j}, I_{i j}\right)_{0 \leq j \leq \mu_{i}} \quad \text { and } \quad S_{i}^{\prime}=\left(S_{i j}^{\prime}, I_{i j}^{\prime}\right)_{0 \leq j \leq \mu_{i}}
$$

are members of $\sigma(R)$ with $\nu_{i}=\mu_{i}-1$ such that $\left(S_{i j}\right)_{0 \leq j \leq \nu_{i}}=\left(S_{i j}^{\prime}\right)_{0 \leq j \leq \nu_{i}}$ is the finite $Q D T$ sequence of $R$ along $V_{i}$. Assume that this double stem is balanced; i.e., it satisfies the condition $\left(\bullet^{*}\right)$

$$
\left(\bigcup_{1 \leq i \leq r}\left\{S_{i \mu_{i}}\right\}\right) \cap\left(\bigcup_{1 \leq i \leq r} Q\left(S_{i \mu_{i}}^{\prime}\right)\right)=\emptyset=\left(\bigcup_{1 \leq i \leq r}\left\{S_{i \mu_{i}}^{\prime}\right\}\right) \cap\left(\bigcup_{1 \leq i \leq r} Q\left(S_{i \mu_{i}}\right)\right)
$$

and the condition which says that

$$
\left\{\begin{array}{l}
\text { by fixing generators } F_{i j} \text { and } F_{i j}^{\prime} \text { of the ideals } I_{i j} \text { and } I_{i j}^{\prime} \text { in } S_{i} \\
\text { and letting } D_{i j}=\operatorname{ord}_{S_{i}} F_{i j} \text { and } D_{i j}^{\prime}=\operatorname{ord}_{S_{i}^{\prime}} F_{i j}^{\prime} \\
\text { we have } D_{i j}=D_{i j}^{\prime} \text { for } 1 \leq i \leq r \text { and } 0 \leq j \leq \nu_{i}
\end{array}\right.
$$

Then upon letting

$$
\mathfrak{a}=\prod_{1 \leq i \leq r} I_{i 0} \quad \text { and } \quad \mathfrak{a}^{\prime}=\prod_{1 \leq i \leq r} I_{i 0}^{\prime}
$$

we have that the ideal $\mathfrak{a}+\mathfrak{a}^{\prime}$ is $M(R)$-primary and

$$
\left(\mathfrak{a}+\mathfrak{a}^{\prime}\right)^{-R}=\prod_{1 \leq i \leq r} \zeta_{R}\left(V_{i}\right)^{D_{i}} \text { where } D_{i}=D_{i \nu_{i}}
$$

with

$$
\mathfrak{D}\left(R, \mathfrak{a}+\mathfrak{a}^{\prime}\right)=\left\{V_{1}, \ldots, V_{r}\right\}
$$


Proof. In view of (b) and Claim (iii) of (3.5), we are done by the proof of $(\ddagger)$ of (3.5).

Theorem 3.7. In the situation of (3.6), replace the assumption of the double stem of $\left(V_{1}, \ldots, V_{r}\right)$ by the assumption of the double arc of $\left(R, V_{1}, \ldots, V_{r}\right)$, by which we mean, for $1 \leq i \leq r$,

$$
S_{i}=\left(S_{i j}, I_{i j}\right)_{0 \leq j \leq \mu_{i}} \quad \text { and } \quad S_{i}^{\prime}=\left(S_{i j}^{\prime}, I_{i j}^{\prime}\right)_{0 \leq j \leq \mu_{i}}
$$

are members of $\sigma\left(R, V_{i}\right)$ with $\nu_{i}=\mu_{i}-1$. Retain the assumption of being balanced. Then the conclusions of (3.6) hold with the supplement that

$$
\left(\mathfrak{a}+\mathfrak{a}^{\prime}\right)^{-R}=\prod_{1 \leq i \leq r} \zeta_{R}\left(V_{i}\right)^{\theta\left(R, S_{i}\right)} .
$$

Proof. Follows from (3.5)(5) and (3.6).

Remark 3.8. (3.4) follows from (3.7) when we observe that replacing $\sigma\left(R, V_{i}\right)$ by $\sigma^{*}\left(R, V_{i}\right)$ amounts to saying that $(3.1) \Rightarrow(3.6)\left(\sharp^{*}\right)$. (3.5)(4)( $\left.\ddagger\right)$ and its proof may be called a local induction since it deals with a single QDT sequence, namely the finite QDT sequence $\left(R_{j}\right)_{0 \leq j \leq \nu}$ of $R$ with $\bar{R}=R_{1}$ and $T=R_{\nu}$. This clever afterthought reminds us of the sentence from the Bhagwat-Gita saying that yoga means doing things cleverly, in Sanskrit: YOGAH KARMASUKAUSHALAM.

Our original proof of (3.4) made a global induction on $\mu=\max \left(\mu_{1}, \ldots, \mu_{r}\right)$. The case of $\mu=1$ follows from $(3.5)(2)(\dagger)$. Now let $\mu>1$ and assume the result is true for all smaller values of $\mu$. Briefly and geometrically speaking, we cut up the curves $\mathfrak{a}, \mathfrak{a}^{\prime}$ into tangential packets $\left(\Delta_{b}\right)_{1 \leq b \leq a}$ according to their initial forms. As in the resolution of singularities described on pages 131-141 of Ab6, this will decompose the "pencil of curves" given by $\mathfrak{a}, \mathfrak{a}^{\prime}$ into members $\left(R_{b}\right)_{1 \leq b \leq a}$ of $Q_{1}(R)$ which, à la Max Noether, represent points in the first neighborhood of the point of the surface mentioned at the end of $(3.5)(3)$. This will strip off the packet $\Delta_{0}$ belonging to $o(R)$. The value of $\mu$ at each $R_{b}$ is smaller than the $\mu$ we started with. End of induction!

In detail, using the notation of (3.3) to (3.5), we restate our goal (3.4.1) as

$$
\left(\mathfrak{a}+\mathfrak{a}^{\prime}\right)^{-R}=\prod_{1 \leq i \leq r} \zeta_{R}\left(V_{i}\right)^{\theta\left(R, S_{i}\right)} .
$$

Let $\Delta_{0}=\left\{i \in\{1, \ldots, r\}: V_{i}=o(R)\right\}$. Let us partition $\{1, \ldots, r\} \backslash \Delta_{0}$ into nonempty disjoint sets $\left(\Delta_{b}\right)_{1 \leq b \leq a}$ according to tangency. Put integers $i, l$ in $\{1, \ldots, r\} \backslash \Delta_{0}$ into the same $\bar{\Delta}_{b}$ iff $S_{i 1}=S_{l 1}$, i.e., geometrically speaking, iff $F_{i 0}$ and $F_{l 0}$ have the same tangent.

For $0 \leq b \leq a$ let $r(b)=\left|\Delta_{b}\right|$ and let $e(b, 1)<e(b, 2)<\cdots<e(b, r(b))$ be the subsequence of $1<2<\cdots<r$ such that $\{e(b, 1), \ldots, e(b, r(b))\}=\Delta_{b}$.

For $1 \leq b \leq a$, upon letting $R_{b}=S_{e(b, 1) 1}$, we get distinct members $\left(R_{b}\right)_{1 \leq b \leq a}$ of $Q_{1}(R)$ and a sequence $\left(V_{e(b, i)}\right)_{1 \leq i \leq r(b)}$ of (not necessarily distinct) members of $D\left(R_{b}\right)^{\Delta}=$ the set of all prime divisors of the two dimensional regular local domain $R_{b}$ with quotient field $L$. 
For $1 \leq b \leq a$, let

$$
\mathfrak{a}_{b}=\prod_{1 \leq i \leq r(b)} I_{e(b, i) 1} \quad \text { and } \quad \mathfrak{a}_{b}^{\prime}=\prod_{1 \leq i \leq r(b)} I_{e(b, i) 1}^{\prime}
$$

and note that these are nonzero nonunit principal ideals in $R_{b}$ such that $\mathfrak{a}_{b}+\mathfrak{a}_{b}^{\prime}$ is $M\left(R_{b}\right)$-primary. For $1 \leq b \leq a$ and $1 \leq i \leq r(b)$, let

$$
\bar{S}_{e(b, i)}=\left(\bar{S}_{e(b, i) j}, \bar{I}_{e(b, i) j}\right)_{0 \leq j \leq \mu(b, i)} \text { and } \bar{S}_{e(b, i)}^{\prime}=\left(\bar{S}_{e(b, i) j}^{\prime}, \bar{I}_{e(b, i) j}^{\prime}\right)_{0 \leq j \leq \mu(b, i)}
$$

be the chopped versions of $S_{e(b, i)}$ and $S_{e(b, i)}^{\prime}$ respectively, where $\mu(b, i)=\mu_{e(b, i)}-1$. Clearly these are members of $\sigma^{*}\left(R_{b}, V_{e(b, i)}\right)$ with $\theta\left(R_{b}, \bar{S}_{e(b, i)}\right)=\theta\left(R_{b}, \bar{S}_{e(b, i)}^{\prime}\right)$.

By $(\bullet)$, for $1 \leq b \leq a$ we get a double curvette

$$
\left(\bar{S}_{e(b, i) j}, \bar{I}_{e(b, i) j}, \bar{S}_{e(b, i) j}^{\prime}, \bar{I}_{e(b, i) j}^{\prime}\right)_{1 \leq i \leq r(b), 0 \leq j \leq \mu(b, i)}
$$

of $\left(R_{b}, V_{e(b, 1)}, \ldots, V_{e(b, r(b)}\right)$, satisfying condition $\left(\bullet_{b}\right)$ obtained from $(\bullet)$ by making an obvious change. For $1 \leq b \leq a$, letting $\mu(b)=\max \{\mu(e(b, 1)), \ldots, \mu(e(b, r(b)))\}$, we clearly have $\mu(b)<\mu$. Therefore by the induction hypothesis, the ideal $\mathfrak{a}_{b}+\mathfrak{a}_{b}^{\prime}$ is $M\left(R_{b}\right)$-primary and we have

$$
\left(\mathfrak{a}_{b}+\mathfrak{a}_{b}^{\prime}\right)^{-R_{b}}=\prod_{1 \leq i \leq r(b)} \zeta_{R_{b}}\left(V_{e(b, i)}\right)^{\theta\left(R_{b}, \bar{S}_{e(b, i)}\right)} .
$$

It can be shown that this equation for $1 \leq b \leq a$ supplemented by $(3.5)(2)(\dagger)$ yields (3.8.1). Since this is only a sketch of an alternative proof of (3.4), we need not give more exhaustive details.

Since $(3.5)(4)(\ddagger)$ is the crux of this entire section, we paraphrase it by observing that in the situation of Theorem (3.6), the main assertion (3.6.1) is equivalent to saying that for any $T \in Q_{l}(R)$ with $l \in \mathbb{N}$ we have

$$
m\left(R, \mathfrak{a}+\mathfrak{a}^{\prime}, T\right)=\sum_{i \in B(T)} D_{i l} \text { where } B(T)=\left\{1 \leq i \leq r: V_{i}=o(T)\right\}
$$

with proof the same as the proof of $(3.5)(4)(\ddagger)$. For a more logical presentation of this section, after reading definition (3.5)(2) of the derived Zariski number $m(R, J, T)$, start with the above version of $(3.5)(4)(\ddagger)$ valid for a balanced double stem which gives rise to Theorem (3.6), then read Theorem (3.7) after reviewing the definition of an arc stem and Basic Fact (3.5)(5), and finally read Theorems (3.1) and (3.4) after looking up the definition of a curvette stem in Section 2.

Note 3.9. As the $r=1$ case of (3.4) supplemented by (3.5)(4), we get the following (which can be "re-proved" by induction on $\mu$ ): For any $V \in D(R)^{\Delta}$ let $S=$ $\left(S_{j}, I_{j}\right)_{0 \leq j \leq \mu}$ and $S^{\prime}=\left(S_{j}^{\prime}, I_{j}^{\prime}\right)_{0 \leq j \leq \mu}$ be any members of $\sigma^{*}(R, V)$ for which we have $S_{\mu} \neq S_{\mu}^{\prime}$ and $\theta(R, S)=\theta\left(R, S^{\prime}\right)$. Then $I_{0}+I_{0}^{\prime}$ is $M(R)$-primary and

$$
\left\{\begin{array}{l}
\operatorname{ord}_{S_{j}} I_{j}=\operatorname{ord}_{S_{j}^{\prime}} I_{j}^{\prime} \text { for } 0 \leq j \leq \mu-1 \\
\text { and }\left(I_{0}+I_{0}^{\prime}\right)^{-R}=\zeta_{R}(V)^{\theta(R, S)} \\
\text { with } \theta(R, S)=d_{\mu-1} \text { and } \mathfrak{D}\left(R, I_{0}+I_{0}^{\prime}\right)=\{V\}
\end{array}\right.
$$




\section{Subideal transforms and tangential maps}

To sharpen the last section, let $R$ be a two dimensional regular local domain with quotient field $L$.

(4A) Subideals. Let $I$ be a nonzero ideal in $R$. For any $T \in Q(R)$, the $(R, T)$ transform $(R, T)(I)$ of $I$ was defined in Section 2 of Ab11. Given any generators $x, y$ of $M(R)$, coefficient set $\kappa$ of $R$, and finite number of nonzero elements $f^{(1)}, \ldots, f^{(p)}$ in $I$, the $(R, x, y, \kappa, T, I)$-transform $(R, x, y, \kappa, T, I)\left(f^{(1)}, \ldots, f^{(p)}\right)$ of $f^{(1)}, \ldots, f^{(p)}$ was defined in Section $(2 \mathrm{~A})$ of Ab13. Let $J$ be any nonzero subideal of $I$, i.e., a nonzero ideal $J$ in $R$ with $J \subset I$. Taking $f^{(1)}, \ldots, f^{(p)}$ to be generators of $J$, we define the $(R, T, I)$-transform of $J$ by putting $(R, T, I)(J)=$ the ideal in $T$ generated by $(R, x, y, \kappa, T, I)\left(f^{(1)}, \ldots, f^{(p)}\right)$. This is easily seen to be independent of the particular generators and the coefficient set.

Let $A=\operatorname{grad}(R)=K[X, Y]$ with $K=R / M(R)$. Let

$$
\bar{P}(A)=\text { set of all nonzero homogeneous principal ideals in } A
$$

and consider the bijection

$$
\epsilon: \kappa[X, Y]^{h m i} \rightarrow \bar{P}^{*}(A)=\bar{P}(A) \cap \operatorname{spec}(A) \text { given by } \epsilon(z)=z A .
$$

Composing $\epsilon^{-1}$ with the tangential bijection $\delta: \kappa[X, Y]^{h m i} \rightarrow Q_{1}(R)$ given in (2B)(3) of [Ab13, we get a bijection

$$
\delta_{R}: \bar{P}^{*}(A) \rightarrow Q_{1}(R) .
$$

We call $\delta_{R}$ the tangential bijection relative to $R$, and for any $\Psi \in \bar{P}^{*}(A)$ we call $\delta_{R}(\Psi)$ the QDT of $R$ in the direction $\Psi$. For any $\Phi \in \bar{P}(A)$ put

$$
\delta_{R}^{\prime}(\Phi)=\left\{\delta_{R}(\Psi): \Psi \in \bar{P}^{*}(A) \text { with } \Phi \subset \Psi\right\} .
$$

We call $\delta_{R}^{\prime}$ the derived tangential map relative to $R$. Note that $\delta_{R}$ and $\delta_{R}^{\prime}$ are clearly independent of the choice of $x, y, \kappa$. Let $d=\operatorname{ord}_{R} I$. By $\mathfrak{f}(R, I)$ we denote the nonzero homogeneous principal ideal in $\operatorname{grad}(R)$ which is the GCD of all the infos of elements of $I$ of $R$-order $d$; we call $\mathfrak{f}(R, I)$ the Zariski gcd of $(R, I)$. By $s(R, I)$ we denote the $(X, Y)$-degree of a generator of $\mathfrak{f}(R, I)$; we call $s(R, I)$ the suborder of $(R, I)$. The Zariski number of $(R, I)$ is defined by putting $m(R, I)=d-s(R, I)$, and for any $T \in Q(R)$ the derived Zariski number is defined by putting $m(R, I, T)=m(T,(R, T)(I))$. If $\operatorname{ord}_{R} J=\operatorname{ord}_{R} I$, then clearly

$$
\mathfrak{f}(R, J) \subset \mathfrak{f}(R, I),
$$

and hence

$$
\mathfrak{f}(R, J)=\mathfrak{f}(R, I) \mathfrak{g}(R, I, J)
$$

for a unique nonzero homogeneous principal ideal $\mathfrak{g}(R, I, J)$ in $\operatorname{grad}(R)$. We call $\mathfrak{g}(R, I, J)$ the reduced gcd of $(R, I, J)$; we put

$$
\mathfrak{P}_{1}(R, I, J)=\delta_{R}^{\prime}(\mathfrak{g}(R, I, J)),
$$

and we call $\mathfrak{P}_{1}(R, I, J)$ the reduced first layer of $(R, I, J)$. Note that if the ideal $J$ is principal, then $\operatorname{info}(R, J)=\mathfrak{f}(R, J)$. Recall that the big stars of $I$ in $R$ are members of $\mathfrak{B}(R, I)=\left\{o_{R}^{-1}(V): V \in \mathfrak{D}(R, I)\right\}=\{T \in \mathfrak{Q}(R, I): m(R, I, T)>0\}$. 
Lemma 4.1. For any nonzero ideal $I$ in $R$ we have

$$
\mathfrak{Q}(R, I) \subset \mathfrak{P}(R, I)
$$

and

$$
\left\{\begin{array}{l}
\mathfrak{Q}(R, I)=\mathfrak{P}(R, I) \\
\Leftrightarrow I=R \text { or } I \text { is } M(R) \text {-primary } \\
\Leftrightarrow(R, T)(I) \text { is } M(T) \text {-primary for all } T \in \mathfrak{P}(R, I) \\
\Leftrightarrow(R, T)(I) \text { is } M(T) \text {-primary for all } T \in \mathfrak{P}_{1}(R, I)
\end{array}\right.
$$

and

$$
\left\{\begin{array}{l}
\text { if } J \text { is any nonzero subideal of } I \text { with } J^{-R}=I^{-R} \text {, then: } \\
J \text { is } M(R) \text {-primary } \Leftrightarrow I \text { is } M(R) \text {-primary }
\end{array}\right.
$$

and

$$
\left\{\begin{array}{l}
\text { if } J \text { is any nonzero subideal of } I \text { with } J^{-R}=I^{-R}, \text { then: } \\
\operatorname{ord}_{R} J=\operatorname{ord}_{R} I \text { with } m(R, J)=m(R, I) \text { and } \\
(R, T)(J)^{-T}=(R, T)(I)^{-T} \text { for all } T \in Q(R) .
\end{array}\right.
$$

Proof. (1) to (3) are easy. (4) follows from Appendix 5 of [ZaS].

Lemma 4.2. Let $J \subset I$ be any nonzero ideals in $R$ with $\operatorname{ord}_{R} J=\operatorname{ord}_{R} I$. Then we have

$$
\delta_{R}^{\prime}(\mathfrak{f}(R, I))=\mathfrak{P}_{1}(R, I) \subset \mathfrak{P}_{1}(R, J)=\delta_{R}^{\prime}(\mathfrak{f}(R, J)) \text { and } m(R, J) \leq m(R, I)
$$

and

$$
(R, T, I)(J)=(R, T)(J) \text { for all } T \in \mathfrak{P}_{1}(R, I)
$$

and

$$
m(R, J)=m(R, I) \Leftrightarrow \mathfrak{f}(R, J)=\mathfrak{f}(R, I) \Rightarrow \mathfrak{P}_{1}(R, J)=\mathfrak{P}_{1}(R, I)
$$

and

$$
\left\{\begin{array}{l}
\text { if }(R, T)(J)^{-T}=(R, T)(I)^{-T} \text { for all } T \in \mathfrak{P}_{1}(R, I) \text { and } \\
\operatorname{ord}_{R} J=\operatorname{ord}_{R} I \text { with } m(R, J)=m(R, I) \text {, then } J^{-R}=I^{-R}
\end{array}\right.
$$

Proof. (1) to (3) are easy. (4) follows from Appendix 5 of [ZaS].

Lemma 4.3. Let $J$ be a nonzero subideal of an $M(R)$-primary ideal $I$ in $R$. Then

$$
J^{-R}=I^{-R} \Leftrightarrow\left\{\begin{array}{l}
\operatorname{ord}_{T}(T,(R, T)(J))=\operatorname{ord}_{T}(T,(R, T)(I)) \text { for all } T \in \mathfrak{Q}(R, I) \\
\text { and } m(R, J, T)=m(R, I, T) \text { for all } T \in \mathfrak{Q}(R, I) .
\end{array}\right.
$$

Proof. Let $h=h(R, I)=$ the dicritical height of $(R, I)$; i.e., $h$ is the unique nonnegative integer such that $\mathfrak{Q}_{h}(R, I) \neq \emptyset=\mathfrak{Q}_{h+1}(R, I)$. For every $j \in \mathbb{N}$, let $l(j)$ be the condition that $(R, T)(J)^{-T}=(R, T)(I)^{-T}$ for all $T \in \mathfrak{Q}_{j}(R, I)$, and let $r(j)$ be the condition that for all $T \in \mathfrak{Q}_{j}(R, I)$ we have $\operatorname{ord}_{T}(T,(R, T)(J))=$ $\operatorname{ord}_{T}(T,(R, T)(I))$ and $m(R, J, T)=m(R, I, T)$. Note that then $l(0)$ is the LHS of (4.3), while the RHS of (4.3) says that $r(j)$ is true for all $j \in \mathbb{N}$. Assuming the RHS of (4.3), by $(4.1)(2)$ and (4.2) we see that for every $j \in \mathbb{N}$ we have $l(j+1) \Rightarrow l(j)$. Since $l(h+1)$ is vacuously true, by decreasing induction on $j$ we get $l(0)$, which is the LHS of (4.3). By (4.1) we see that the LHS of (4.3) implies the RHS of (4.3). 
Lemma 4.4. Let $I$ be any $M(R)$-primary ideal in $R$, let $\mathfrak{a}$ and $\mathfrak{b}$ be any nonzero principal subideals of $I$, and for every $T \in Q(R)$ let

$$
I_{T}=(R, T)(I) \text { with } \mathfrak{a}_{T}=(R, T, I)(\mathfrak{a}) \text { and } \mathfrak{b}_{T}=(R, T, I)(\mathfrak{b}) .
$$

Then we have the following.

(4.4.1). Let $T \in \mathfrak{Q}(R, I)$ be such that $\min \left(\operatorname{ord}_{T} \mathfrak{a}_{T}, \operatorname{ord}_{T} \mathfrak{b}_{T}\right)=\operatorname{ord}_{T} I_{T}$ and $m\left(T, I_{T}\right) \neq 0$. Then

$$
m\left(T, \mathfrak{a}_{T}+\mathfrak{b}_{T}\right)=m\left(T, I_{T}\right) \Leftrightarrow\left\{\begin{array}{l}
\operatorname{ord}_{T} \mathfrak{a}_{T}=\operatorname{ord}_{T} I_{T}=\operatorname{ord}_{T} \mathfrak{b}_{T} \text { with } \\
\mathfrak{P}_{1}\left(T, I_{T}, \mathfrak{a}_{T}\right) \cap \mathfrak{P}_{1}\left(T, I_{T}, \mathfrak{b}_{T}\right)=\emptyset .
\end{array}\right.
$$

(4.4.2). We have

$$
(\mathfrak{a}+\mathfrak{b})^{-R}=I^{-R} \Leftrightarrow\left\{\begin{array}{l}
\min \left(\operatorname{ord}_{T} \mathfrak{a}_{T}, \operatorname{ord}_{T} \mathfrak{b}_{T}\right)=\operatorname{ord}_{T} I_{T} \text { for all } T \in \mathfrak{Q}(R, I), \\
\operatorname{and} m\left(T, \mathfrak{a}_{T}+\mathfrak{b}_{T}\right)=m\left(T, I_{T}\right) \text { for all } T \in \mathfrak{B}(R, I) .
\end{array}\right.
$$

Proof. To prove (4.4.1) we note that $\mathfrak{a}_{T}$ and $\mathfrak{b}_{T}$ are nonzero principal subideals of the $M(T)$-primary ideal $I_{T}$ in the two dimensional regular local domain $T$. Hence the general case follows from the special case when $T=R$.

In the special case, upon letting $d=\operatorname{ord}_{R} I$ with $s=s(R, I)$, we are assuming that $\min \left(\operatorname{ord}_{R} \mathfrak{a}, \operatorname{ord}_{R} \mathfrak{b}\right)=d$ with $d-s>0$, and we want to show that

$$
m(R, \mathfrak{a}+\mathfrak{b})=d-s \Leftrightarrow \operatorname{ord}_{R} \mathfrak{a}=d=\operatorname{ord}_{R} \mathfrak{b} \text { and } \mathfrak{P}_{1}(R, I, \mathfrak{a}) \cap \mathfrak{P}_{1}(R, I, \mathfrak{b})=\emptyset .
$$

If $m(R, \mathfrak{a}+\mathfrak{b})>0$, then clearly $\operatorname{ord}_{R} \mathfrak{a}=d=\operatorname{ord}_{R} \mathfrak{b}$. Consequently, assuming that $\operatorname{ord}_{R} \mathfrak{a}=d=\operatorname{ord}_{R} \mathfrak{b}$, it suffices to show that

$$
m(R, \mathfrak{a}+\mathfrak{b})=d-s \Leftrightarrow \mathfrak{P}_{1}(R, I, \mathfrak{a}) \cap \mathfrak{P}_{1}(R, I, \mathfrak{b})=\emptyset .
$$

Now

$$
\operatorname{info}(R, \mathfrak{a})=\mathfrak{f}(R, I) \mathfrak{g}(R, I, \mathfrak{a}) \text { and } \operatorname{info}(R, \mathfrak{b})=\mathfrak{f}(R, I) \mathfrak{g}(R, I, \mathfrak{b})
$$

and hence

$$
m(R, \mathfrak{a}+\mathfrak{b})=d-s \Leftrightarrow \operatorname{GCD}(\mathfrak{g}(R, I, \mathfrak{a}), \mathfrak{g}(R, I, \mathfrak{b}))=1
$$

and clearly

$$
\operatorname{GCD}(\mathfrak{g}(R, I, \mathfrak{a}), \mathfrak{g}(R, I, \mathfrak{b}))=1 \Leftrightarrow \mathfrak{P}_{1}(R, I, \mathfrak{a}) \cap \mathfrak{P}_{1}(R, I, \mathfrak{b})=\emptyset .
$$

This proves (4.4.1). By taking $J=\mathfrak{a}+\mathfrak{b}$ in (4.2) and (4.3), we get (4.4.2).

With this preparation at hand, let us sharpen Theorem (3.6) by proving:

Theorem 4.5. Let $\left(V_{1}, \ldots, V_{r}\right)$ be any finite sequence of (not necessarily distinct) members of $D(R)^{\Delta}$ with $r \in \mathbb{N}_{+}$and consider the $M(R)$-primary complete ideal I in $R$ given by

$$
I=\prod_{1 \leq i \leq r} \zeta_{R}\left(V_{i}\right)^{n(i)} \quad \text { with } \quad n(i) \in \mathbb{N}_{+} .
$$

Note that letting $\left(S_{i j}\right)_{0 \leq j \leq \nu_{i}}$ be the finite QDT sequence of $R$ along $V_{i}$, we have

$$
\mathfrak{Q}(R, I)=\left\{S_{i j}: 1 \leq i \leq r \text { and } 0 \leq j \leq \nu_{i}\right\} \text { and } \mathfrak{B}(R, I)=\left\{S_{i \nu_{i}}: 1 \leq i \leq r\right\} .
$$

Given any nonzero principal subideals $\mathfrak{a}$ and $\mathfrak{b}$ of $I$, for every $T \in Q(R)$ let

$$
I_{T}=(R, T)(I) \text { with } \mathfrak{a}_{T}=(R, T, I)(\mathfrak{a}) \text { and } \mathfrak{b}_{T}=(R, T, I)(\mathfrak{b}) .
$$


Then

$$
(\mathfrak{a}+\mathfrak{b})^{-R}=I \Leftrightarrow\left\{\begin{array}{l}
\min \left(\operatorname{ord}_{T} \mathfrak{a}_{T}, \operatorname{ord}_{T} \mathfrak{b}_{T}\right)=\operatorname{ord}_{T} I_{T} \text { for all } T \in \mathfrak{Q}(R, I) \\
\text { and } \operatorname{ord}_{T} \mathfrak{a}_{T}=\operatorname{ord}_{T} \mathfrak{b}_{T} \text { for all } T \in \mathfrak{B}(R, I), \\
\text { and } \mathfrak{P}_{1}\left(T, I_{T}, \mathfrak{a}_{T}\right) \cap \mathfrak{P}_{1}\left(T, I_{T}, \mathfrak{b}_{T}\right)=\emptyset \text { for all } T \in \mathfrak{B}(R, I)
\end{array}\right.
$$

Proof. Follows from (3.5)(2)(b) and (4.4).

\section{REFERENCES}

[Ab1] Shreeram Abhyankar, On the valuations centered in a local domain, Amer. J. Math. 78 (1956), 321-348. MR0082477 (18,556b)

[Ab2] Shreeram Abhyankar, Ramification theoretic methods in algebraic geometry, Annals of Mathematics Studies, no. 43, Princeton University Press, Princeton, N.J., 1959. MR.0105416(21 \#4158)

[Ab3] S. S. Abhyankar, Resolution of singularities of embedded algebraic surfaces, 2nd ed., Springer Monographs in Mathematics, Springer-Verlag, Berlin, 1998. MR.1617523 (99c:14021)

[Ab4] Shreeram S. Abhyankar, Historical ramblings in algebraic geometry and related algebra, Amer. Math. Monthly 83 (1976), no. 6, 409-448. MR0401754 (53 \#5581)

[Ab5] Shreeram S. Abhyankar, Desingularization of plane curves, Singularities, Part 1 (Arcata, Calif., 1981), Proc. Sympos. Pure Math., vol. 40, Amer. Math. Soc., Providence, RI, 1983, pp. 1-45. MR713043 (85d:14024)

[Ab6] Shreeram S. Abhyankar, Algebraic geometry for scientists and engineers, Mathematical Surveys and Monographs, vol. 35, American Mathematical Society, Providence, RI, 1990. MR.1075991 (92a:14001)

[Ab7] Shreeram S. Abhyankar, Polynomial expansion, Proc. Amer. Math. Soc. 126 (1998), no. 6, 1583-1596, DOI 10.1090/S0002-9939-98-04183-5. MR:1443142 (98g:12003)

[Ab8] S. S. Abhyankar, Lectures on Algebra I, World Scientific, 2006. MR2253320 (2007k:13001)

[Ab9] Shreeram S. Abhyankar, Inversion and invariance of characteristic terms: Part I, The legacy of Alladi Ramakrishnan in the mathematical sciences, Springer, New York, 2010, pp. 93-168, DOI 10.1007/978-1-4419-6263-8_8. MR2744259(2012b:14057)

[Ab10] Shreeram S. Abhyankar, Dicritical divisors and Jacobian problem, Indian J. Pure Appl. Math. 41 (2010), no. 1, 77-97, DOI 10.1007/s13226-010-0017-x. MR.2650101 (2011d:14107)

[Ab11] Shreeram S. Abhyankar, Pillars and towers of quadratic transformations, Proc. Amer. Math. Soc. 139 (2011), no. 9, 3067-3082, DOI 10.1090/S0002-9939-2011-10731-7. MR.2811263 (2012f:14003)

[Ab12] Shreeram S. Abhyankar, More about dicriticals, Proc. Amer. Math. Soc. 139 (2011), no. 9, 3083-3097, DOI 10.1090/S0002-9939-2011-10732-9. MR2811264 (2012d:13047)

[Ab13] Shreeram S. Abhyankar, Quadratic transforms inside their generic incarnations, Proc. Amer. Math. Soc. 140 (2012), no. 12, 4111-4126, DOI 10.1090/S0002-9939-2012-11583-7. MR.2957201

[Ab14] S. S. Abhyankar, Dicritical divisors and Dedekind's Gauss lemma, Proc. Amer. Math. Soc., to appear.

[AH1] Shreeram S. Abhyankar and William J. Heinzer, Existence of dicritical divisors, Amer. J. Math. 134 (2012), no. 1, 171-192, DOI 10.1353/ajm.2012.0002. MR2876143

[AH2] Shreeram S. Abhyankar and William J. Heinzer, Existence of dicritical divisors revisited, Proc. Indian Acad. Sci. Math. Sci. 121 (2011), no. 3, 267-290, DOI 10.1007/s12044-0110035-6. MR2867982(2012h:14148)

[AL1] Shreeram S. Abhyankar and Ignacio Luengo, Algebraic theory of dicritical divisors, Amer. J. Math. 133 (2011), no. 6, 1713-1732, DOI 10.1353/ajm.2011.0045. MR2863374

[AL2] S. S. Abhyankar and I. Luengo, Spiders and dicriticals, Proc. Amer. Math. Soc., to appear.

[Gor] P. Gordon, Invariantentheorie, Leipzig, 1885.

[Kle] F. Klein, Entwiklung der Mathematik, Berlin, 1926. 
[Nag] Masayoshi Nagata, Local rings, Interscience Tracts in Pure and Applied Mathematics, No. 13, Interscience Publishers, a division of John Wiley \& Sons, New York-London, 1962. MR0155856 (27 \#5790)

[Noe] Max Noether, Zur Theorie des eindeutigen Entsprechens algebraischer Gebilde von beliebig vielen Dimensionen, Math. Ann. 2 (1870), no. 2, 293-316, DOI 10.1007/BF01444023 (German). MR1509663

[Sal] G. Salmon, Higher Plane Curves, Dublin, 1852.

[ZaS] Oscar Zariski and Pierre Samuel, Commutative algebra. Vol. II, Springer-Verlag, New York, 1975. Reprint of the 1960 edition; Graduate Texts in Mathematics, Vol. 29. MR0389876(52 \#10706)

Department of Mathematics, Purdue University, West Lafayette, Indiana 47907

E-mail address: ram@cs.purdue.edu

Departamento de Matemáticas-IUma, Universidad Zaragoza, Calle de Pedro CerBUNA 12, E-50009, ZARAgOZA, SPAIN

E-mail address: artal@unizar.es 